PAPER • OPEN ACCESS

Novel technique for estimating pollution-associated leakage current in high-voltage insulators based on adaptive filtering in long-term recording

To cite this article: Jose-M Bueno-Barrachina et al 2021 Meas. Sci. Technol. 32055105

View the article online for updates and enhancements. 


\title{
Novel technique for estimating pollution-associated leakage current in high-voltage insulators based on adaptive filtering in long-term recording
}

\author{
Jose-M Bueno-Barrachina ${ }^{1}(\mathbb{D})$, César-S Cañas-Peñuelas ${ }^{1}$ (i), Yiyao Ye-Lin ${ }^{2}$ (i) \\ and Vicente Fuster-Roig ${ }^{1}$ (D) \\ ${ }^{1}$ Instituto de Tecnología Eléctrica, Universitat Politècnica de València, 46022 Valencia, Spain \\ ${ }^{2}$ Centro de Investigación e Innovación en Bioingeniería, Universitat Politècnica de València, 46022 \\ Valencia, Spain \\ E-mail: yiye@eln.upv.es
}

Received 22 October 2020, revised 1 February 2021

Accepted for publication 26 February 2021

Published 25 March 2021

\begin{abstract}
Moist polluting substances on high-voltage insulator surfaces can cause power-line failures by triggering electric arcs. There are at present no effective methods of measuring insulator pollution levels during normal operations. In this work, we attempt to estimate insulator pollution leakage current (PLC) as an indirect method of measuring deposits in a 30 month period of simultaneously recording leakage current and related environmental variables in substation insulators. We analyzed the relationship between raw leakage current and environmental variables. We canceled out the influence of relative humidity on leakage current by adaptive filtering and then obtained the PLC by filtering out the anomalous peaks in the recording. The proposed method considerably reduces the correlation between leakage current and relative humidity $(0.826$ vs 0.019$)$. The resulting signal was only slightly correlated with other environmental variables $(<0.03)$, suggesting that the relationships between leakage current and temperature, wind direction and speed are mainly attributable to their influence on relative humidity. The PLC presents a quasi-annual smooth fluctuation over time with a magnitude similar to those obtained in laboratory tests. This novel technique could be used to monitor insulator PLC in real time and thus improve power supply continuity and reduce maintenance costs.
\end{abstract}

Keywords: leakage current, flashover, high-voltage insulator, adaptive filtering, pollution deposits associated with leakage current, pollution leakage current

(Some figures may appear in colour only in the online journal)

\section{Introduction}

High-voltage electrical insulators in outdoor substations are routinely exposed to polluting substances such as dust, of the Creative Commons Attribution 4.0 licence. Any further distribution of this work must maintain attribution to the author(s) and the title of the work, journal citation and DOI. chemicals, traffic pollution, and mineral salts at coastal substations, which accumulate on exposed surfaces forming contamination deposits. When the insulator surface is dry, the leakage current due to ohmic conduction is negligible. However, when combined with the moisture caused by light rain, fog, mist, or dew, it can form a conductive layer on the surface and trigger an electric arc through the well-known pollution flashover process $[1,2]$ in which leakage current begins to flow through this wet conductive layer and heats the surface. Over an extended period of time this process may cause the electric 
field to increase locally due to the appearance of dry areas (dry bands [3]), and it may initiate partial discharges that finally trigger an electric arc [3], which can irreversibly damage the insulator coating and cause power-line failure. Although this behavior can be substantially improved by insulation coating, as the coat ages, hydrophobicity is reduced and the dry band increases, producing subsequent partial discharges.

To guarantee power system continuity and quality, some research groups have focussed on determining the reliable performance limits of the electric insulators during normal operations, analyzing the different causes of leakage currents. Some attempted to analyze the optimal insulator shape and different surface treatments to minimize pollution deposits associated with leakage current (PLC), while others aimed to determine the threshold level of soluble or non-soluble polluting substances that trigger the dry band in order to prevent electric $\operatorname{arcs}[4-8]$.

Previous studies have used lab tests to determine the relationship between the deposit levels of soluble and non-soluble salts $[9,10]$ and leakage currents under predefined working conditions $[11,12]$ to obtain valuable information on the risk of electric arc onset. However, in real applications, a mixture of sediments whose composition varies over time is deposited on the insulator surface [9], which makes it difficult to determine the PLC level and its possible negative consequences on the insulator. There is currently no effective method available to directly or indirectly measure pollution deposits in real-time applications, which would be very useful for electrical companies in conducting maintenance [11] or monitoring the state of insulator coatings (e.g., room temperature vulcanization, RTV) and their useful life.

Monitoring insulator PLC could be used to indirectly measure the contamination deposit level due to the underlying relationship between these two factors. Abeysekara et al analyzed the leakage current in glass insulators for different saline deposit levels and found that the kurtosis value of the leakage current increased with saline deposition $(k=2.93$ for clean insulators vs $k=12.33$ and 22.11 for 10 and $40 \mathrm{~g} \mathrm{l}^{-1}$ saline deposits, respectively) [11]. The superficial resistance of the insulator was found to be inversely proportional to the ESDD [13], while Fierro-Chavez reported that the ESDD was proportional to the leakage current $[14,15]$. Sahli et al proposed a multiple regression model to estimate insulator surface conductivity from different pollution compositions, obtaining an $R^{2}$ of $99 \%$ [16]. Other authors have also reported that the pollution level greatly influenced the leakage current and led to a lower flashover voltage [16-18].

Salem et al [19, 20] and Chandrasekar et al [21] attempted to estimate the pollution deposits on the insulator surface by analyzing harmonic distortion in the $150-600 \mathrm{~Hz}$ bandwidth of a leakage current waveform obtained in laboratory tests. However, this technique still presents some technical difficulties in measuring pollution deposits in real-time applications. This is due to the fact that high-voltage lines themselves usually have a large component of harmonic distortion, which may be associated with the use of nonlinear loads by the end users, such as electronic power devices using current in abrupt short pulses [22]. It is therefore difficult to detect harmonic distortions due to pollution deposits from those associated with the network itself during normal operations. In this context, the root-mean-square value of leakage current is a more reliable measurement since it is less influenced by harmonic distortion. Even so, insulator leakage current measurements in real-time applications depend not only on pollution deposits but also on environmental variables, which fluctuate over time $[10,23]$. Salam et al found that the leakage current increased as the wind speed increased in the range of 2-4 $\mathrm{m} \mathrm{s}^{-1}$ [24] and other authors found that the leakage current increased when insulators lost their hydrophobicity [25-29]. Several studies have reported the relationship between leakage current and relative humidity [13, 25, 27], and others found that temperature changes had a minor effect on leakage currents [30]. In summary, no techniques have so far been reported to estimate PLC during normal insulator operations in actual service.

We here propose a novel technique to estimate PLC in outdoor insulators in real time during normal operations by eliminating the influence of environmental variables, improving power supply continuity and quality, and minimizing unexpected power-line failures. We validate and demonstrate its effectiveness in a 30 month long-term recording of leakage current and related environmental variables in substation insulators. Section 2 describes the materials and data analysis method. Section 3 demonstrates its effectiveness in canceling out the influence of environmental variables on leakage current and analyzes the characteristics of the remainder leakage current. Section 4 contains a discussion of the origin of the remainder leakage current and outlines the limitations of this work.

\section{Materials and methods}

\subsection{Test setup}

The study analyzed the in-situ performance of seven new insulators installed on a test panel in an outdoor substation on the Mediterranean coast with a high level of saline contamination. Their main characteristics were shown in table 1 .

Insulators 1-6 were the same substation post-porcelain type with different silicone RTV coatings, except number 3 without a coating. Number 7 was a silicone composite-line insulator. All the insulators were connected to the same phase of a $20 \mathrm{kV}$ busbar (figure 1)

Figure 2 shows a block diagram of the experimental setup. The isolators to be tested were mounted on another auxiliary isolator on the pole structure to confine the leakage currents to the measurement system. To measure both the surface and capacitive current, which vary according to the pollution deposits, an insulated copper cable was connected to the junction between both insulators, through which the leakage current flowed to the measurement system. The insulated copper cable was connected to a plate with shunt resistors protected from possible short-circuit currents to the ground by a surge arrester, the measurement ranges being from $600 \mu \mathrm{A}$ to $4000 \mu \mathrm{A}$.

The voltage in the resistor terminals was processed by a signal conditioning circuit with a 4-20 mA current loop output, 


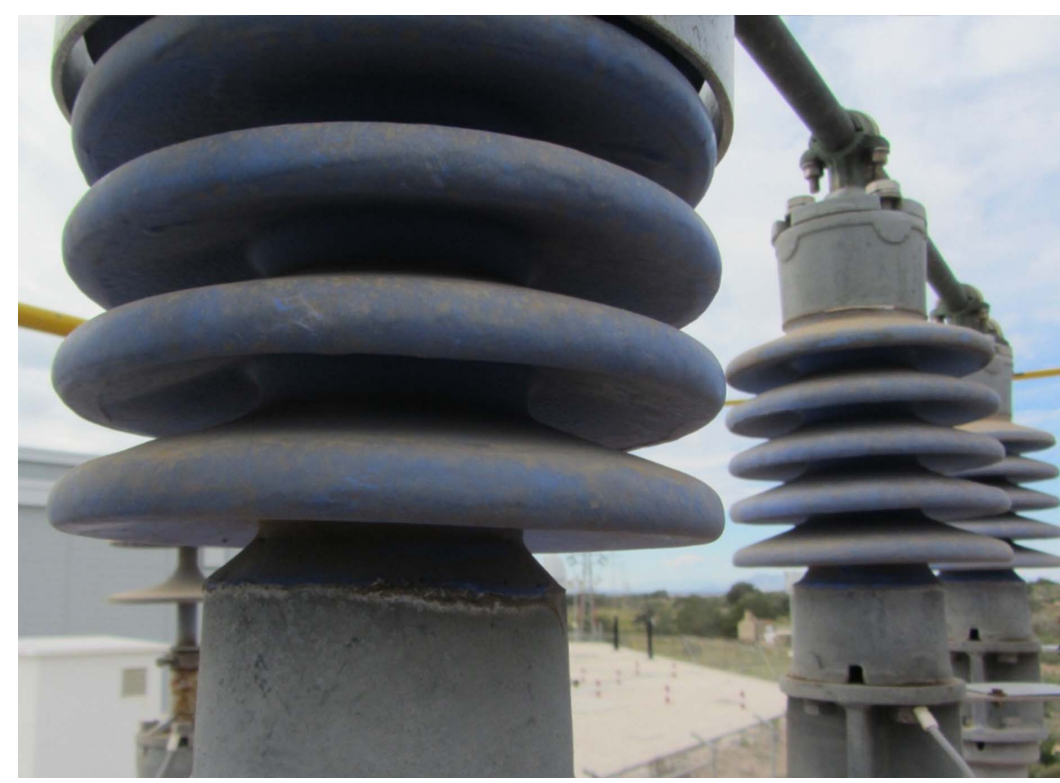

Figure 1. Coated insulators in the test panel.

\section{$20 \mathrm{kV}$ BUSBAR}
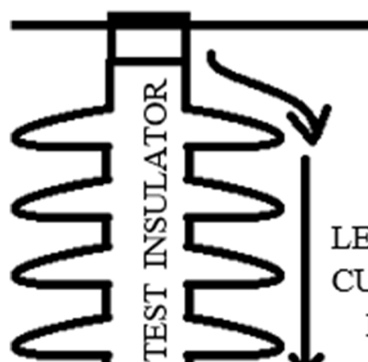

LEAKAGE

CURRENT

PATH

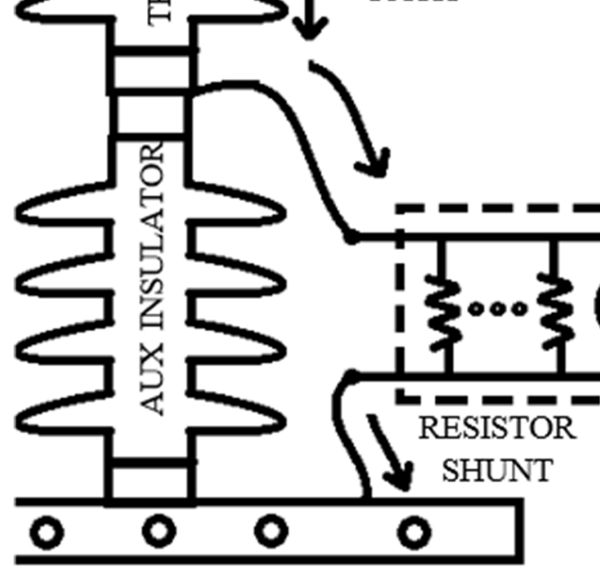

POLE STRUCTURE

Figure 2. Sketch of the experimental setup.

which was electrically isolated to protect the rest of the measurement equipment. The root-mean-square value was computed continuously every $250 \mathrm{~ms}$, taking into account the bandwidth of the target signal $(<800 \mathrm{~Hz})$. The 4-20 mA current loop was connected to the input of a CR $1000 \times$ commercial data logger specially designed for extreme outdoor conditions (Campbell Scientific Company). The average value of
Table 1. Characteristics of tested insulators.

\begin{tabular}{|c|c|c|}
\hline $\begin{array}{l}\text { Insulator } \\
\text { number }\end{array}$ & $\begin{array}{l}\text { Insulator } \\
\text { base material }\end{array}$ & Insulator coating \\
\hline 1 & Porcelain & $\begin{array}{l}\text { A type (Medium silicone proportion }+ \\
\text { filler) manufacture } 1\end{array}$ \\
\hline 2 & Porcelain & $\begin{array}{l}\text { B type (High silicone proportion }+ \\
\text { filler) }\end{array}$ \\
\hline 3 & Porcelain & Without coating \\
\hline 4 & Porcelain & $\begin{array}{l}\text { C type (Medium silicone proportion }+ \\
\text { filler) manufacture } 2\end{array}$ \\
\hline 5 & Porcelain & $\begin{array}{l}\text { D type (Medium silicone proportion }+ \\
\text { filler) manufacture } 3\end{array}$ \\
\hline 6 & Porcelain & $\begin{array}{l}\text { E type (Medium silicone proportion }+ \\
\text { filler) manufacture } 4\end{array}$ \\
\hline 7 & $\begin{array}{l}\text { Silicone } \\
\text { composite }\end{array}$ & - \\
\hline
\end{tabular}

the signal was obtained in 5 min windows and stored on a microSD card using a CR Basic programming language software for further processing to reduce the computational cost.

In addition to the leakage current in the seven insulators, the environmental variables were also recorded, including temperature, relative humidity, and wind direction and speed at a rate of one sample every $5 \mathrm{~min}$ by means of a METSENS600 meteorological station, which is compatible and easily integrated with any Campbell Scientific data logger through an SDI-12 port. The study period extended from February 2015 to August 2017.

\subsection{Data analysis}

The Spearman correlation coefficient, which measures the strength and direction of monotonic nonlinear relationships between two ranked variables, was computed for the environmental variables to assess their underlying non-linear 


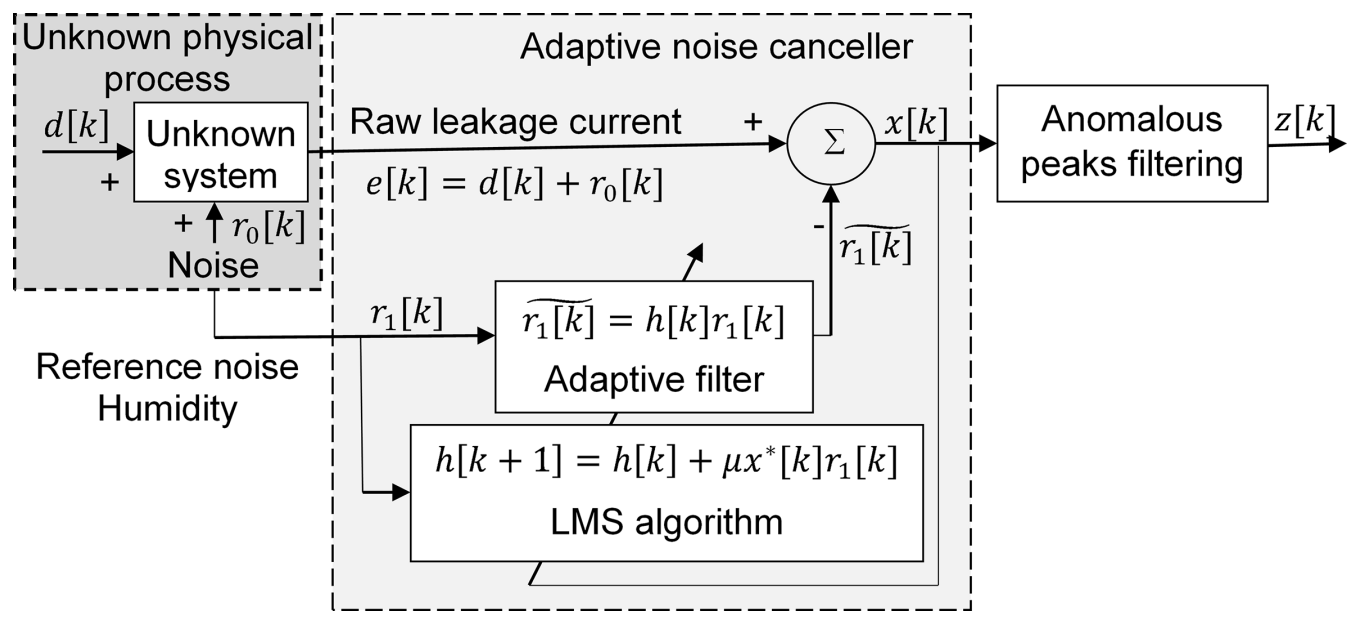

Figure 3. Data analysis flowchart to estimate the pollution-related leakage current.

relationship with leakage current. As there may be a relationship between the different variables that could mask a relationship with leakage current [31, 32], to determine these variables' intrinsic relationship with leakage current we also computed the partial Spearman correlation coefficient between raw leakage current and the environmental variables, which obtained a moderate average correlation above 0.20 when controlling the effect of the other variables. For example, when we analyze the partial correlation of leakage current with wind speed, it may indicate the relationship between these variables by removing possible humidity-wind speed, wind direction-wind speed and temperature-wind speed interactions. Least-squares adjustments were also carried out to determine the underlying relationship between the raw leakage current and the different variables using the lineal, exponential and second-order polynomial functions.

We then attempted to cancel out the influence of relative humidity on leakage current, since a close correlation between these two factors may be expected [33]. In this context, the conventional filter is ineffective since the bandwidth of the relative humidity signal is unknown and can vary over time. We assume that the raw leakage current $e[k]$ is an unknown mixture of relative humidity, modulated leakage current $r_{0}[k]$, pollution leakage current and others $d[k]$. In this context, $r_{0}[k]$, which represents the variation of the leakage current associated with relative humidity, could be expected to correlate with the recorded relative humidity $\left(r_{1}[k]\right)$. We therefore decided to use adaptive filtering to cancel out the influence of relative humidity on leakage current using the recorded relative humidity as a reference noise signal $r_{1}[k]$ (see figure 3 ). An adaptive filter was used to estimate the noise source $\widetilde{r_{1}[k]}=h[k] r_{1}[k]$. The system output of the adaptive noise canceler $x[k]$ was the source estimation, which is the target signal $d[k]$ plus noise source $r_{0}[k]$, and then subtracted from the estimated noise source $r_{1}[k]$. If $r_{0}[k]$ and $r_{1}[k]$ are close enough, a better estimation of the target signal can be obtained. For this, the system output $x[k]$ has to be minimized in terms of the LMS of the power as follows:

$$
x[k]=d[k]+r_{0}[k]-\widetilde{r_{1}[k]}
$$

Squaring equation (1) and considering the condition that the signal source $d[k]$ is uncorrelated with the noise signal, $r_{0}[k]$ and $\widetilde{r_{1}[k]}$ produce

$$
E\left[x^{2}\right]=E\left[d^{2}\right]+E\left[\left(r_{0}-\widetilde{r_{1}}\right)^{2}\right]
$$

The best least square estimate of the signal $\mathrm{d}[\mathrm{k}]$ can be achieved by minimizing $E\left[\left(r_{0}-\widetilde{r_{1}}\right)^{2}\right]$ in the least square mean.

$$
E\left[x^{2}\right]=E\left[d^{2}\right]
$$

The least mean square (LMS) algorithm is used to obtain a desired filter by finding the filter coefficients that attempt to produce the LMS of the system output $x[k]$. The filter coefficient is usually initialized at zero, and updated for each sample as follows:

$$
h[k+1]=h[k]+\mu x^{*}[k] \cdot r_{1}[k]
$$

where $h[k]=\left(h_{0}[k], h_{1}[k], \ldots h_{p-1}[k-p+1]\right)^{\mathrm{T}}$ is the filter coefficient in sample $k, p$ is the filter order, and $\mu$ is step size. We established a filter order to 20 and step size $\mu=10^{-6}$.

After canceling out the influence of relative humidity on the leakage current, anomalous current peaks are usually present in the signal output $x[k]$. These anomalous peaks may be associated with other external factors [34], for example, power cuts or extreme adverse environmental conditions, such as rain and frost. We proceeded to eliminate these anomalous current peaks by means of a specially designed filter based on the target signal information. These usually presented much higher amplitudes than PLC, which should present smooth but not sudden variations over time. This filter consisted of two phases: an initial phase in which the adaptive threshold level Threshold $_{1}[n]$ was applied to a 1 month moving window with $50 \%$ overlap. The average of the threshold levels of the last 

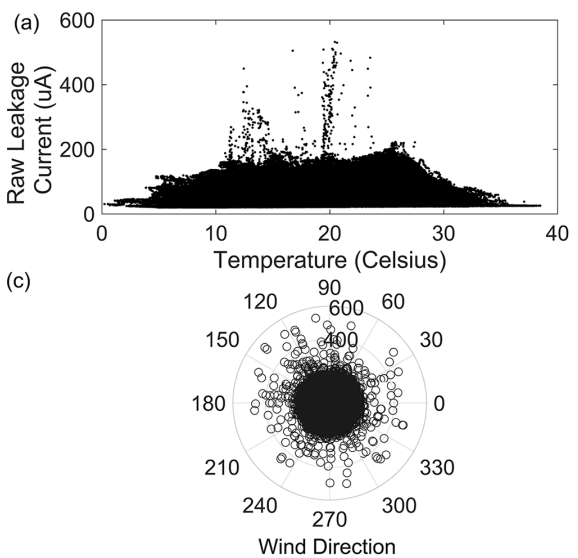
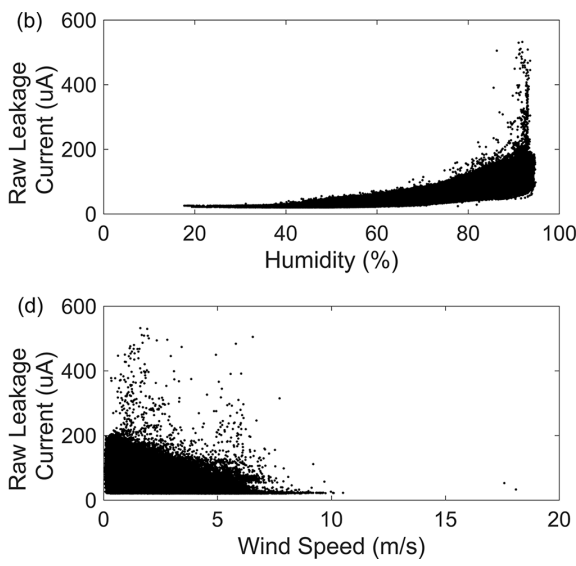

Figure 4. Scatter representation of raw leakage current and the different environmental variables. (a) Temperature. (b) Relative humidity. (c) Wind direction. (d) Wind speed.

three analysis windows was calculated and the resulting signal was labeled $y[k]$.

$$
\begin{gathered}
\operatorname{Threshold}_{1}[n]=\frac{\operatorname{Thr}[n]+\operatorname{Thr}[n-1]+\operatorname{Thr}[n-2]}{3} \\
\operatorname{Thr}[n]=\mu[n] \pm b \cdot \sigma[n]=\mu[n] \pm 2 \sigma[n]
\end{gathered}
$$

where $\operatorname{Thr}[n], \operatorname{Thr}[n-1]$ and $\operatorname{Thr}[n-2]$ were the threshold levels of the last three analysis windows, which were calculated from the mean $\mu$ and standard deviation $\sigma$ of the signal $x[k]$ of the analysis window. Both the window length (1 month), the number of windows to consider, and the coefficient $b$ were experimentally determined to cancel out the anomalous peaks as far as possible using a conservative criterion while keeping the target information.

In the second phase, we attempted to eliminate the current peaks that were not canceled out in the previous phase due to the high density of anomalous variations over time. Based on the fact that the PLC varies gradually over time, the cumulative 2-point derived function was implemented to accentuate the abrupt variation of the leakage current (see equation (7)). The resulting signal was squared to further accentuate the abrupt leakage current variation. A second adaptive threshold level Threshold $_{2}[n]$ was finally applied for the moving window of this latter signal $H[k]$, thus obtaining the remainder leakage current $z[k]$.

$$
\begin{gathered}
H[k]=(|y[k]-y[k-1]|+|y[k-1]-y[k-2]|)^{2} \\
\operatorname{Threshold}_{2}[n]=\mu[n] \pm c \cdot \sigma[n]
\end{gathered}
$$

where $y[\mathrm{k}]$ was the output signal of the first phase of the anomalous peaks filter, $\mu[n]$ and $\sigma[n]$ the mean and standard deviation of signal $H[k]$ in the analysis window. In this case, the best result was obtained for a 2 month moving window with a $50 \%$ overlap. Unlike the previous phase, the threshold level was calculated only with the statistics of the current analysis window without taking into account the previous analysis windows. The coefficient $c$ was experimentally determined for each insulator to cancel out the abnormal current peaks as far as possible. Again, we used a conservative criterion to establish this coefficient $c$ while preserving the target information.

The effectiveness of eliminating the influence of environmental variables was assessed by calculating the Spearman correlation coefficient of the rectified remainder leakage current $|z[k]|$ of each insulator with different environmental variables to compare with those obtained for raw leakage current.

We also attempted to compare the remaining leakage currents of different insulators to determine their susceptibility to accumulating polluting substances by subtracting both the mean, standard deviation and maximum value from the rectified signal of the remainder leakage current $|z[k]|$.

\section{Results}

Figure 4 shows the scatter plot of the raw leakage current of insulator 3 and the different environmental variables. The raw leakage current presented a weak non-linear relationship with temperature (see trace a), with the lowest value for both high and low temperatures $\left(T>30{ }^{\circ} \mathrm{C}\right.$ or $\left.T<5{ }^{\circ} \mathrm{C}\right)$. No prevailing wind direction was obtained throughout the whole year (see trace c), which had no clear relationship with raw leakage current; there was a clear non-linear relationship between raw leakage current and humidity (trace b) and wind speed (trace d); high wind speeds were associated with low leakage current while high humidity was accompanied by high leakage current values.

Table 2 shows the insulators' Spearman correlation coefficient of raw leakage current with different environmental variables. Figure 5 gives the average $R^{2}$ value of the least-squares adjustments between them for the seven insulators. Different insulators had similar relationships with different environmental variables. The raw leakage current barely correlated with temperature, achieving a relatively low average correlation coefficient of $0.139 \pm 0.063$. This was also reflected in a low average $R^{2}$ (below 0.05 ) regardless of the adjustment function type, while it was strongly related to humidity, regardless of insulator type, with an average correlation coefficient of up to $0.826 \pm 0.051$. In this case, the polynomial and exponential 
Table 2. Spearman correlation coefficient of raw leakage current with different environmental variables. Partial correlation was obtained for raw leakage current and humidity, wind direction and speed when the effect of the other variables was controlled.

\begin{tabular}{|c|c|c|c|c|c|c|c|c|}
\hline \multirow[b]{2}{*}{ Insulator } & \multicolumn{2}{|c|}{ Temperature } & \multicolumn{2}{|c|}{ Humidity } & \multicolumn{2}{|c|}{ Wind direction } & \multicolumn{2}{|c|}{ Wind speed } \\
\hline & Corr & Partial & Corr & Partial & Corr & Partial & Corr & Partial \\
\hline 1 & 0.114 & NA & 0.860 & 0.870 & -0.315 & -0.070 & -0.494 & -0.142 \\
\hline 2 & 0.085 & NA & 0.858 & 0.853 & -0.311 & -0.073 & -0.490 & -0.115 \\
\hline 3 & 0.275 & NA & 0.717 & 0.750 & -0.334 & -0.115 & -0.397 & -0.117 \\
\hline 4 & 0.116 & NA & 0.847 & 0.851 & -0.315 & -0.077 & -0.487 & -0.134 \\
\hline 6 & 0.121 & NA & 0.849 & 0.857 & -0.313 & -0.068 & -0.487 & -0.135 \\
\hline 7 & 0.114 & NA & 0.810 & 0.801 & -0.303 & -0.063 & -0.447 & -0.066 \\
\hline$\mu \pm \sigma$ & $0.139 \pm 0.063$ & NA & $0.826 \pm 0.051$ & $0.834 \pm 0.043$ & $-0.315 \pm 0.009$ & $-0.076 \pm 0.018$ & $-0.469 \pm 0.036$ & $-0.123 \pm 0.029$ \\
\hline
\end{tabular}

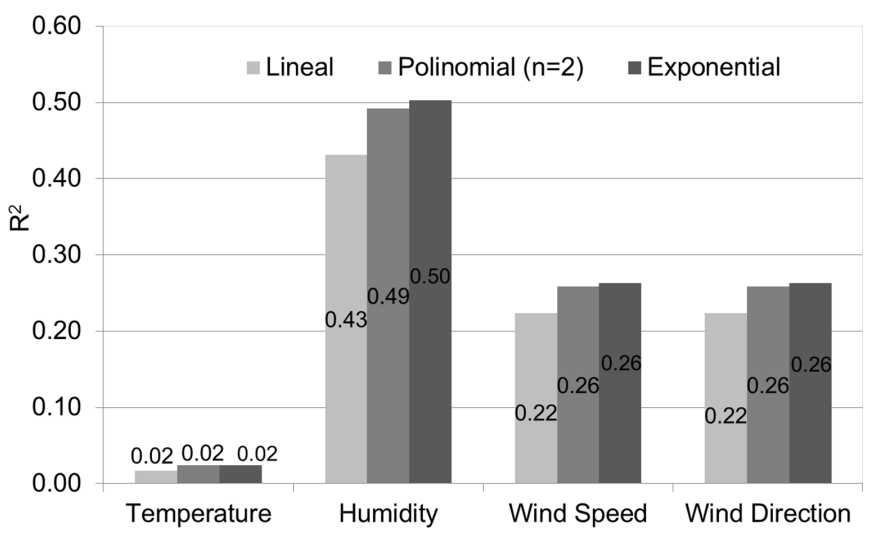

Figure 5. Average $R^{2}$ value of least squares adjustments between raw leakage current with different environment variables for the seven insulators.

functions better described the underlying relationship than the linear function, obtaining an average $R^{2}$ of about 0.50 . Raw leakage current also presented a moderate inverse relationship with wind direction and speed, obtaining an average correlation coefficient of $-0.315 \pm 0.009$ and $-0.469 \pm 0.036$, respectively. Both the polynomial and exponential functions were found to better define the underlying relationship of raw leakage current with wind direction and speed $\left(R^{2} \sim 0.25\right)$. We therefore further analyzed the partial spearman correlation of the raw leakage current of individual insulators with humidity, wind direction and speed while controlling the effects of the other variables. As can be seen in table 2, the raw leakage current had a high partial correlation with humidity $(0.834 \pm 0.043)$ and a considerably reduced partial correlation with wind direction and speed of $-0.076 \pm 0.018$ and $-0.123 \pm 0.029$, respectively.

Figure 6 shows an example of the simultaneous recording of relative humidity (trace a) and raw leakage current (trace b). Trace $\mathrm{c}$ shows the corresponding $x[k]$ after eliminating the influence of relative humidity by an adaptive filter. It can be seen that the time evolution of both raw leakage current and relative humidity signals show a similar trend, which disappears after applying the adaptive filter. In addition, the proposed custom-designed filter also appropriately filters out the anomalous peaks embedded in the original recording, obtaining a remaining leakage current $\mid z, k] \mid$ with a regular variation over time. This latter presents a quasi-annual fluctuation over time, achieving the minimum value in winter and the maximum value in summer.

Unlike raw leakage current, we did not find any specific relationship of the rectified remainder leakage current $|z[k]|$ with any environmental variable (see scatter plot in figure 7). Table 3 shows the Spearman correlation coefficient of the rectified remainder leakage current $|z[k]|$ with different environmental variables. Again, regardless of insulator type, $|z[k]|$ after eliminating the influence of humidity and anomalous peaks, it presents an irrelevant correlation with different environmental variables below 0.03 .

The representative statistical data of the rectified remainder leakage current are shown in table 4. The remainder leakage current of the insulator 3 was about 10 times higher than the others, obtaining an average leakage current of $9.53 \pm 10.253 \mu \mathrm{A}$. The behavior of insulators 1,4 and 5 was similar, with an average leakage current of about $0.9 \mu \mathrm{A}$. The best performance with the lowest value was obtained for insulator 7 , with an average leakage current of $0.275 \pm 0.298 \mu \mathrm{A}$.

\section{Discussion}

In this work, we attempted to estimate insulator PLC by eliminating the influence of environmental variables. For this, we first analyzed the underlying relationship between the recorded raw leakage current and different environmental variables. Raw leakage current was found to be closely correlated with relative humidity, the model that best describes this relationship being an exponential or polynomial function. This finding agrees with that of Zhicheng et al, who analyzed the relationship between leakage current and relative humidity for different levels of polluting substances in lab tests and confirmed that this latter obeyed an exponential function [6]. In comparison to insulator 3 without coating, the raw leakage current measured on RTV-coated insulators presents a higher correlation with relative humidity. This is probably due to the fact that uncoated insulators have a more heterogeneous distribution of water droplets on the insulator surface in the presence of humidity, giving rise to different current circulation paths, so that the leakage current magnitude obtained is more dispersed. On the other hand, the leakage current magnitude in 
(a)

(b)
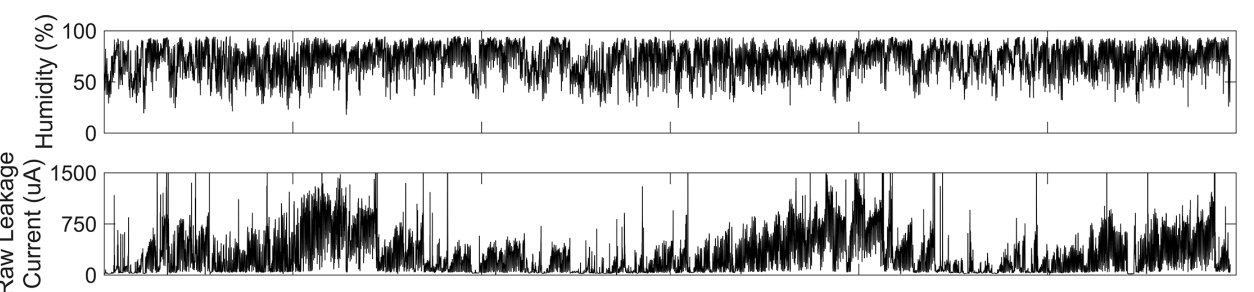

(c)

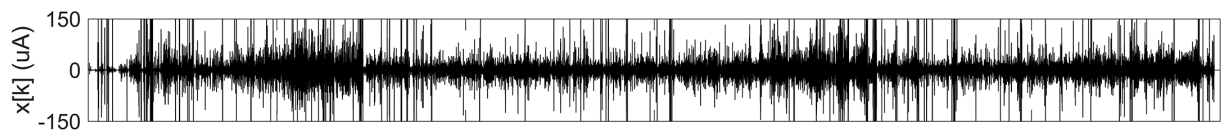

(d)

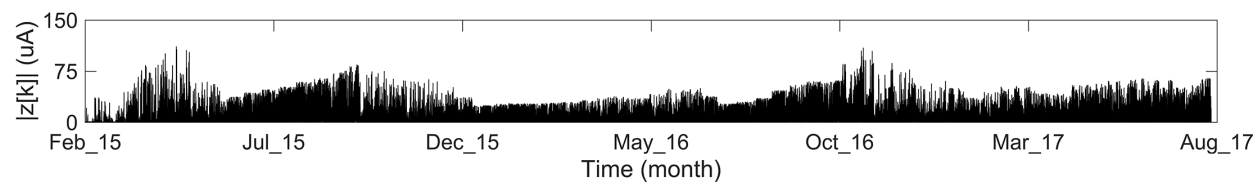

Figure 6. Simultaneous recording of relative humidity (a) and raw leakage current (b) of insulator 3 after cancelling out relative humidity by adaptive filtering (c) and after filtering out the anomalous peaks in the recording (d).
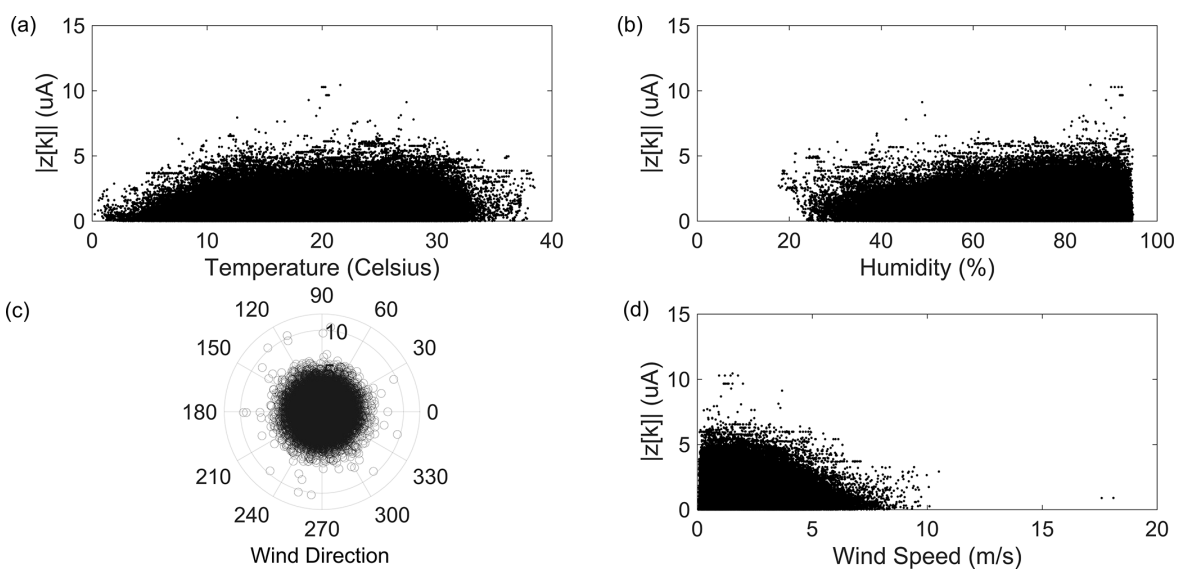

Figure 7. Scatter representation between the rectified signal of remainder leakage current $|z[k]|$ and the different environmental variables: (a) temperature. (b) Relative humidity. (c) Wind direction. (d) Wind speed.

Table 3. Spearman correlation coefficient of processed data $z[k]$ with different environmental variables.

\begin{tabular}{lcclc}
\hline Insulator & Temperature & Humidity & Wind direction & Wind speed \\
\hline 1 & 0.015 & 0.021 & -0.027 & 0.008 \\
2 & 0.034 & 0.001 & -0.017 & 0.004 \\
3 & -0.009 & 0.034 & -0.023 & 0.041 \\
4 & 0.000 & 0.032 & -0.021 & -0.012 \\
5 & 0.013 & 0.023 & -0.025 & -0.004 \\
6 & 0.014 & 0.024 & -0.025 & -0.012 \\
7 & 0.008 & -0.001 & -0.010 & 0.027 \\
$\mu \pm \sigma$ & $0.011 \pm 0.013$ & $0.019 \pm 0.014$ & $-0.021 \pm 0.006$ & $0.007 \pm 0.020$ \\
\hline
\end{tabular}

insulators with RTV coating is more predictable, due to the more homogeneous distribution of drops on the surface, giving rise to a greater correlation with relative humidity.

In addition, we found that low raw leakage current values were obtained for both the high- and low-temperature ranges, giving rise to a weak correlation between these two factors. This finding partially agrees with other authors, who reported that leakage current decreased due to drying as temperature increased [30]. Nevertheless, we also found low leakage current values at temperatures below $5{ }^{\circ} \mathrm{C}$, which may be associated with the specific weather conditions of the installation. Salam found that wind direction may give rise to the heterogeneous deposition of polluting substances on insulator surfaces [17], while Hussain found increased saline deposits in the prevailing wind direction in short time periods [18]. Both distance from the sea and exposure time influenced insulator saline deposits [18]. In this work, we did not find the prevailing wind direction, since the long-term recording lasted for only 30 months. However, we did find a moderate inverse relationship between raw leakage current and wind direction, 
Table 4. Representative statistical data ( $\mu \pm \sigma$ and maximum) of the rectified remainder leakage current of the different insulators.

\begin{tabular}{llr}
\hline Insulator & $\mu \pm \sigma(\mu \mathrm{A})$ & Maximum $(\mu \mathrm{A})$ \\
\hline 1 & $0.876 \pm 0.896$ & 10.439 \\
2 & $0.479 \pm 0.503$ & 7.790 \\
3 & $9.530 \pm 10.253$ & 111.517 \\
4 & $0.922 \pm 0.979$ & 11.153 \\
5 & $0.997 \pm 1.089$ & 12.391 \\
6 & $0.634 \pm 0.669$ & 6.097 \\
7 & $0.275 \pm 0.298$ & 3.712 \\
\hline
\end{tabular}

possibly due to the specific orientation with respect to the sea. Specifically, a small angle in the wind direction was associated with moist saline deposits and higher leakage current values. On the other hand, wind speed had a moderate inverse relationship with leakage current, which disagrees with Salam et al, who reported higher leakage current at high wind speeds in their lab tests and described this relationship as $I=D_{\mathrm{c}}(w v)^{b}$ [17]. This discrepancy may be due to the fact that they only analyzed the influence of wind speed in a restricted range of $2-4 \mathrm{~m} \mathrm{~s}^{-1}$ in their tests, and we have focussed on the analysis of real-world long-term data. Our results partially agree with Hussain et al, who reported a non-linear relationship between leakage current and wind speed [18]. They observed that leakage current increased with wind speeds below $8 \mathrm{~m} \mathrm{~s}^{-1}$ due to higher pollution deposits on insulator surfaces. By contrast, leakage current tended to decrease at wind speeds above $8 \mathrm{~m} \mathrm{~s}^{-1}$, due to the drying effect [18]. Our results suggest that the relationship between raw leakage current and environmental variables throughout the year in long-term recordings is much more complex than in short-term recording or lab conditions. To our knowledge, this is the first analysis of real-world long-term data and paves the way for a better understanding of the underlying interactions between raw leakage current and different environmental variables.

Indeed, we found that the partial correlation with wind direction and speed when controlling the effect of other environmental variables was much weaker than their corresponding correlation coefficients. In addition, the remainder leakage current after canceling the influence of relative humidity on leakage current presented a correlation coefficient with the different environmental variables of less than 0.03 . These results suggest that the relationship between leakage current and temperature, wind direction and speed were mainly attributable to the relative humidity fluctuation produced by the variation of these environmental variables, indicating that it is not necessary to design a specific filter to cancel out the influence of these three variables on leakage current, as we did for relative humidity. Our results agree with those of Castillo Sierra, who found that temperature and wind direction only caused a small change in the risk of failure [31, 32]. We also agree with Salam et al, who stated that temperature changes had a negligible effect on leakage current at constant relative humidity in lab tests [17]. Our findings also agree with Hussain $e t a l$, who reported that the non-linear relationship between leakage current and wind speed was associated with the wetting and drying process, and therefore with relative humidity [18]. This finding considerably simplifies the required data processing for estimating PLC and also reduces the associated cost, since it becomes unnecessary to measure temperature, wind direction and speed.

In this work, we proposed to use adaptive filtering to cancel the influence of relative humidity, using the recorded relative humidity as the reference noise signal. Using the adaptive threshold levels by simple statistical methods, the anomalous peaks filtering could effectively filter out both sparse and high-density (in time) anomalous current peaks. We believe that the rectified remainder leakage current $|z[k]|$ was associated with polluting substances for a number of reasons. First, the rectified remainder leakage current was not attributable to the different environmental variables, since their correlation was negligible (see table 3). Second, a similar trend was obtained for the remainder leakage current of the different insulators, although we do not show this data for reasons of brevity. All of these presented a quasi-annual fluctuation, which may be due to the self-cleaning effect of the weather conditions. Moreover, the remainder leakage current magnitude of the different insulators was found to be within the PLC range obtained in the laboratory. In lab tests carried out on a $14 \mathrm{kV}$ generator applied to a glass insulator with no pollution, Bezerra et al obtained an average maximum leakage current of $8.5 \mu \mathrm{A}$ and $12 \mu \mathrm{A}$ respectively [35]. When an equivalent salt deposit density $(\mathrm{ESDD})=0.2 \mathrm{mg} \mathrm{cm}^{-2}$ pollution and suspension conductivity $=80 \mathrm{mS} \mathrm{cm}^{-1}$ was applied, an average maximum leakage current of $42.5 \mu \mathrm{A}$ and $60 \mu \mathrm{A}$ was obtained, respectively [35]. These results are compatible with those we obtained for insulator 3 (average and maximum leakage current of 9.530 and $111.517 \mu \mathrm{A}$, respectively). When applying a $19 \mathrm{kV}$ voltage in lab tests, an average leakage current of about $6.5 \mu \mathrm{A}$ was obtained, before the premature aging process of the silicone composite insulator tested [36], which is similar to that obtained for insulator 7 in table 4 . Roman et al measured the leakage current in in-situ silicon composite insulators in a high-voltage direct current (HVDC) transmission line (400-500 kV) and obtained long-term values ranging from 10 to $60 \mu \mathrm{A}$ [37]. The leakage current variability obtained in these studies may be associated with different test conditions, e.g., different applied voltages. In addition, we also performed real outdoor long-term recordings rather than laboratory data, which may give rise to different leakage current magnitudes due to an unknown pollution composition.

Despite the promising results, this work is not exempt from limitations. First, considering that the useful life of an insulator [38, 39] varies between 16 and 30 years and the average life is around 24 years, the materials aging during their first 2 years of life can be considered as negligible. Nevertheless, the remainder leakage current in long term recordings in real applications contains not only the PLC but also the fluctuation of leakage current due to aging. Future work should determine the feasibility of measuring PLC and material aging effects. In practice, it will not be necessary to determine aging and pollution deposits separately to improve insulator maintenance. When the remainder leakage current is close to the critical value of leakage currents, suggesting a failure risk [31], an installation stop can be programmed to clean the insulators. 
Increasing the frequency of installation stops would be related to the material aging effect, so that the old insulators would have to be replaced by new ones. Second, we did not conduct a discrete (one sample for each spaced time) measurement of pollution deposits to further validate our method and provide an experimental curve of PLC magnitude and pollution deposits. In this respect, we would like to highlight the difficulty of measuring pollution deposits in real applications. In addition, measuring pollution deposits by cleaning the insulator may alter the sample. Consequently, we will not be able to determine the real evolution of pollution deposits on the surface and the possible effect of natural cleaning.

To our knowledge, this is the first study to report a novel technique for estimating PLC that can be easily implemented in real-time applications, which is fundamental for keeping insulators below the critical leakage current in real time. This could be very helpful for better quantifying insulator performance in-situ, enhancing power supply continuity and quality by avoiding power failures caused by electrical arcing and optimizing human resources for high-voltage line maintenance. Since the proposed method allows canceling out the influence of environmental variables on the leakage current, it will not be necessary to differentiate dry and wet periods to determine the risk failure, as in Castillo Sierra et al [31, 40]. This technique has the added advantage that no preliminary information is required on relative humidity fluctuations, but only an additional sensor for measuring this magnitude. We therefore believe that this technique could be used for all types of substation insulators, regardless of their geographical location, although we only validated it in a specific substation. The cost of this novel technique is relatively low, since it only requires the installation of the relative humidity and leakage current sensor on insulators to predict their leakage current. With the latest advances in electronic instrumentation, the relative humidity sensor could be further integrated into the leakage current sensor itself without an excessive additional cost. In addition, a microcontroller, microprocessor or digital signal processor with the proposed algorithm should be included for data processing. It would also be recommended to establish a training period of at least 3 months to collect enough data to compute the statistical measures to determine the optimal threshold to filter out the anomalous current peaks from recordings in individual substations. After estimating the PLC by the proposed method, this information could be transmitted in real time for efficient planning of insulation maintenance.

\section{Conclusions}

In this work, we analyzed the underlying relationship between long-term raw insulator leakage current data and different environmental variables, and found that the relationship between raw leakage current and temperature, wind direction and speed was due to the influence of these variables on relative humidity. We also proposed a novel technique to estimate PLC by eliminating the influence of relative humidity by means of adaptive filtering. Specific anomalous peak filtering was designed based on adaptive threshold levels by simple statistical methods to effectively filter out both sparse and high-density (in time) anomalous peaks in the recording, thereby obtaining the PLC with gradual variations over time. The proposed technique is low-cost and paves the way for estimating PLC in real time, which would better determine the performance of different insulators in situ, improving power-line continuity and quality. At the same time, this would also facilitate planning scheduled power-line shutdowns, lead to better resource management for conducting the associated tasks, and consequently reduce maintenance costs.

\section{ORCID iDs}

Jose-M Bueno-Barrachina (D) https://orcid.org/0000-00017191-8220

César-S Cañas-Peñuelas (D) https://orcid.org/0000-00024781-3151

Yiyao Ye-Lin (D) https://orcid.org/0000-0003-2929-181X

Vicente Fuster-Roig (D) https://orcid.org/0000-0002-24289203

\section{References}

[1] Gençoğlu M T and Cebeci M 2008 The pollution flashover on high voltage insulators Electr. Power Syst. Res. 78 1914-21

[2] Rahal A and Huraux C 1979 Flashover mechanism of high voltage insulators IEEE Trans. Power Appar. Syst. PAS-98 2223-31

[3] Alston L L and Zoledziowski S 1963 Growth of discharges on polluted insulation Proc. Inst. Electr. Eng. 110 1260-6

[4] Arshad A, Nekahi A, McMeekin S G and Farzaneh M 2018 Measurement of surface resistance of silicone rubber sheets under polluted and dry band conditions Electr. Eng. 100 1729-38

[5] Venkataraman S and Gorur R S 2006 Prediction of flashover voltage of non-ceramic insulators under contaminated conditions IEEE Trans. Dielectr. Electr. Insul. 13 862-9

[6] Zhicheng G, Yingke M, Liming W, Ruihai L, Hua W and Yi M 2009 Leakage current and discharge phenomenon of outdoor insulators Int. J. Electr. Eng. Inf. 1 1-17

[7] Li J, Sima W, Sun C and Sebo S A 2010 Use of leakage currents of insulators to determine the stage characteristics of the flashover process and contamination level prediction IEEE Trans. Dielectr. Electr. Insul. 17 490-501

[8] Li J Y, Sun C X, Sima W X and Yang Q 2009 Stage pre-warning based on leakage current characteristics before contamination flashover of porcelain and glass insulators IET Gener. Transm. Distrib. 3 605-15

[9] Ramos N G, Campillo R M T and Naito K 1993 A study on the characteristics of various conductive contaminants accumulated on high voltage insulators IEEE Trans. Power Deliv. 8 1842-50

[10] Schindelholz E and Kelly R G 2012 Wetting phenomena and time of wetness in atmospheric corrosion: a review Corros. Rev. 30 135-70

[11] Abeysekara A H A D, Kumara J R S S, Fernando M A R M, Eakanayake M P B, Godaliyadda G M R I and Wijayakulasooriya J V 2017 Remote leakage current detector for identification of insulators discharges IEEE Trans. Dielectr. Electr. Insul. 24 2449-58

[12] Gouda O E and El Dein A Z 2014 Experimental techniques to simulate naturally polluted high voltage transmission line insulators IEEE Trans. Dielectr. Electr. Insul. 21 2199-205 
[13] Amin M and Salman M 2006 Aging of polymeric insulators (an overview) Rev. Adv. Mater. Sci. 13 93-116

[14] Fierro-Chavez J L, Ramirez-Vazquez I and Montoya-Tena G 1996 On-line leakage current monitoring of $400 \mathrm{kV}$ insulator strings in polluted areas IEE Proc. Gener. Transm. Distrib. 143 560-4

[15] Ramirez-Vazquez I and Fierro-Chavez J L 1999 Criteria for the diagnostic of polluted ceramic insulators based on the leakage current monitoring technique Conf. on Electrical Insulation and Dielectric Phenomena (CEIDP), Annual Report pp 715-8

[16] Sahli Z, Mekhaldi A, Boudissa R and Boudrahem S 2011 Prediction parameters of dimensioning of insulators under non-uniform contaminated conditions by multiple regression analysis Electr. Power Syst. Res. 81 821-9

[17] Salam M A, El-fadil N and Nadir Z 2006 A novel approach to predict leakage current in insulators from wind velocity ECTI Trans. Electr. Eng. Electron. Commun. 4 47-50

[18] Hussain M M, Chaudhary M A and Razaq A 2019 Mechanism of saline deposition and surface flashover on high-voltage insulators near shoreline: mathematical models and experimental validations Energies 12 1-20

[19] Salem A A, Abd-Rahman R, Al-Gailani S A, Kamarudin M S, Ahmad H and Salam Z 2020 The leakage current components as a diagnostic tool to estimate contamination level on high voltage insulators IEEE Access 8 92514-28

[20] Salem A A, Abd-Rahman R, Al-Gailani S A, Salam Z, Kamarudin M S, Zainuddin H and Yousof M F M 2020 Risk assessment of polluted glass insulator using leakage current index under different operating conditions IEEE Access 8 175827-39

[21] Chandrasekar S, Kalaivanan C and Karthikeyan S 2008 Study on pollution severity of porcelain insulators using LC and phase angle measurement 2008 IEEE Region 10 and the Third Int. Conf. Industrial and Information Systems (IEEE) pp 1-5

[22] Albadi M H, Al Abri R S, Al Hinai A S and Al-Badi A H 2018 Harmonics temporal profile in high-voltage networks: case study Power System Harmonics: Analysis, Effects and Mitigation Solutions for Power Quality Improvement ed Z Ahmed, H E Shady, A Abdel and E B Murat (London: Intechopen Limited) pp 35-50

[23] Amin M, Amin S and Ali M 2009 Monitoring of leakage current for composite insulators and electrical devices Rev. Adv. Mater. Sci. 21 75-89

[24] Salam M A, Nadir Z and El-fadil N 2006 Characterization of flashover voltage of a polluted insulator energized with DC voltage ECTI Trans. Electr. Eng. Electron. Commun. 4 51-5

[25] Nakanishi T, Komiya H, Shinokubo H, Matsuoka R, Kumagai S, Hikita M and Irie T 2002 Comparative leakage current measurements on silicone rubber rods in salt fog chamber by different measuring systems Conf. Record of IEEE Int. Symp. Electrical Insulation pp 252-5

[26] Lambrecht J, Barsch R, Gubanski S M and Fernando M A R M 1998 Evaluation of the hydrophobicity-dynamics of artificially polluted silicone-rubber model insulators CSD'98. Proc. 1998 IEEE 6th Int. Conf. Conduction and Breakdown in Solid Dielectrics (Cat. No.98CH36132) pp 430-3

[27] Matsuoka R, Naito K, Irie T and Kondo K 2002 Evaluation methods of polymer insulators under contaminated conditions IEEE/PES Transmission and Distribution Conf. and Exhibition vol 3 pp 2197-202

[28] Ito A, Marungsri B, Satake A, Shinokubo H, Matsuoka R, Guo Z J and Yu Z 2003 Fundamental investigation results of diagnostic techniques for deteriorated polymer insulators Proc. 7th Int. Conf. Properties and Applications of Dielectric Materials (Cat. No.03CH37417) vol 1 pp 385-8

[29] Sampe T et al 2004 Fundamental investigation results of diagnostic technique for deteriorated polymer insulators Conf. Record of the 2004 IEEE Int. Symp. Electrical Insulation vol 1 pp 288-91

[30] Ahmad A S, Ghosh P S, Aljunid S A K and Ahmad S 2003 Estimation of leakage current level on the transformer bushing using regression technique Proc. 7th Int. Conf. Properties and Applications of Dielectric Materials (Cat. No.03CH37417) vol 3 pp 1174-7

[31] Castillo Sierra R, Oviedo-Trespalacios O, Candelo J E and Soto J D 2015 Assessment of the risk of failure of high voltage substations due to environmental conditions and pollution on insulators Environ. Sci. Pollut. Res. 22 9749-58

[32] Castillo Sierra R, Oviedo-Trespalacios O, Candelo J E and Soto J D 2015 The influence of atmospheric conditions on the leakage current of ceramic insulators on the Colombian Caribbean coast Environ. Sci. Pollut. Res. 22 2526-36

[33] Mei H W, Mao Y K, Bian X M, Peng G M, Wang L M and Guan Z C 2010 Effect of relative humidity on the maximum leakage current of insulators Gaodianya Jishu/High Volt. Eng. 36 627-31

[34] Salem A A and Abd-rahman R 2018 A review of the dynamic modelling of pollution flashover on high voltage outdoor insulators J. Phys. Conf. Ser. 1049 1-11

[35] de Barros Bezerra J M, Lima A M N, Deep G S and da Costa E G 2009 An evaluation of alternative techniques for monitoring insulator pollution IEEE Trans. Power Deliv. 24 1773-80

[36] Fernando R M and Gubanski S M 2010 Ageing of silicone rubber insulators in coastal and inland tropical environment IEEE Trans. Dielectr. Electr. Insul. 17 326-33

[37] Roman M, Van Zyl R R, Parus N and Mahatho N 2019 In-situ monitoring of leakage current on composite and glass insulators of the cahora bassa HVDC transmission line SAIEE Africa Res. J. 110 4-10

[38] Amin M and Amin S 2014 Aging research on sir and tpe insulators (an overview) Rev. Adv. Mater. Sci. 36 29-39

[39] Ghosh D and Khastgir D 2018 Degradation and stability of polymeric high-voltage insulators and prediction of their service life through environmental and accelerated aging processes ACS Omega 3 11317-30

[40] Chrzan K L, Vosloo W L and Holtzhausen J P 2011 Leakage current on porcelain and silicone insulators under sea or light industrial pollution IEEE Trans. Power Deliv. 26 2051-2 